\title{
Parity of Pions and CP Violation in Neutral Kaon System
}

\author{
Brian Robson \\ Department of Theoretical Physics, Research School of Physics and Engineering \\ The Australian National University, Canberra, ACT 0200, Australia \\ E-mail: Brian.Robsoneanu. edu. au
}

\begin{abstract}
The parity of pions is discussed within the framework of the Generation Model of particle physics and it is shown that both the 1954 determination of the parity of the charged pions and the 2008 determination of the parity of the neutral pion are compatible with the mixed-parity nature of the pions predicted by a recent composite Generation Model. The development of the Generation Model as an alternative to the Standard Model of particle physics is discussed. It is demonstrated how the Generation Model leads to a unified classification of leptons and quarks and how this makes feasible a composite model of the fundamental particles of the Standard Model. In particular it is shown that the $1964 C P$ violating experiment of Christenson et al. may be understood without $C P$ violation.
\end{abstract}

36th International Conference on High Energy Physics

July 4-11, 2012

Melbourne, Australia 


\section{Introduction}

This paper summarizes the Generation Model (GM) of particle physics, which has been developed over the last decade [1-4]. Essentially, the GM is obtained from the Standard Model (SM) [5] by making two postulates, which together maintain the same transition probabilities for both hadronic and leptonic processes as the SM so that agreement with experiment is preserved.

The differences between the GM and the SM lead to several new paradigms in particle physics: strange quarks in nucleons [3]; origin of mass [4,6,7]; origin of gravity [4,6,7] and mixed-parity quark states in hadrons $[3,4,8-10]$. This paper shows that the GM provides an understanding of charge-conjugation-parity $(C P)$ symmetry in the decay of the long-lived neutral kaon, which is absent in the SM.

Section 2 demonstrates that the intrinsic parity of pions played a decisive role in the overthrow of both parity conservation in 1957 and $C P$ conservation in 1964 within the framework of the SM. Section 3 indicates the basic problem inherent in the SM. Section 4 introduces the GM and indicates how the simpler and unified GM classification scheme overcomes the problems inherent in the SM's classification of the elementary matter particles of the SM in terms of additive quantum numbers. Finally section 5 states the conclusion that the mixed-parity nature of the charged pions allows the observed decay of the long-lived neutral kaon into two charged pions without $C P$ violation.

\section{Parity of Pions in Standard Model}

In the SM pions are assumed to have parity $P=-1$. This value was first obtained in 1954 by Chinowsky and Steinberger [11] using the capture of negatively charged pions by deuterium to form two neutrons: $\pi^{-}+D \rightarrow 2 n$. This preceded the quark model so that in the analysis of the experiment, the pion, the proton and the neutron were each assumed to be an elementary particle with no substructure.

Following the adoption of the quark model as part of the SM, the parity of the pions remained accepted as $P=-1$. In the quark model, the proposed combinations of up and down quarks and their antiparticles for the three pions are such that if the quarks have intrinsic parity $P=+1$ while the corresponding antiquarks have intrinsic parity $P=-1$, all the three pions have parity $P=-1$, in agreement with the result of Chinowsky and Steinberger.

This parity of the pions led to the overthrow of both parity conservation and $C P$ conservation in weak interaction processes. We shall now describe briefly how this came about.

\subsection{Tau-Theta Puzzle}

In the period 1947-53 several new particles were discovered. In particular one charged meson, named the tau meson, decayed to three charged pions, while another charged meson, named the theta meson, decayed to two pions: $\tau^{+} \rightarrow \pi^{+}+\pi^{+}+\pi^{-}, \theta^{+} \rightarrow \pi^{+}+\pi^{0}$. Analysis of the 
decays of both these particles indicated that they had closely similar lifetimes and masses. These properties suggested that the tau and theta particles were simply two different decay modes of the same particle.

In 1953-54 Dalitz suggested that a study of the energy distribution of the three pions in tau meson decays would provide information about the spin and parity of the tau meson. Such analyses, assuming $P=-1$ for pions, led to the conclusion that the tau meson had $J^{P}=0^{-}$or $2^{-}$. On the other hand, the two-body decay of the theta meson gave the opposite parity for the two even spin values, indicating that the tau and theta mesons were different particles.

This tau-theta puzzle was resolved in 1956 by Lee and Yang, who suggested that parity was violated in weak interactions. This suggestion was rapidly confirmed in 1957 in three independent experiments. These experiments indicated that the tau and theta mesons were indeed the same meson (now called the $K^{+}$) and also showed that charge-conjugation $(C)$ was also violated in weak interactions.

\subsection{Neutral Kaon Mixing}

In 1955 Gell-Mann and Pais considered the behavior of neutral particles under the chargeconjugation operator. In particular they considered the neutral kaon and realized that unlike the photon and the neutral pion, which transform into themselves under the $C$ operator so that they are their own antiparticles, the antiparticle of the neutral kaon, which has strangeness $S=+1$, was a distinct particle, since it had a different strangeness quantum number: $\mathrm{S}=-1$.

They concluded that the two neutral mesons, the kaon and its antiparticle, are degenerate particles that exhibit unusual properties, since they can transform into each other via weak interactions through intermediate two-pion states.

In order to treat this novel situation, Gell-Mann and Pais suggested that it was more convenient to employ different particle states, rather than the neutral kaon and its antiparticle, to describe neutral kaon decay. They suggested that these particle states be quantum mechanical mixtures of the sum $\left(K_{1}^{0}\right)$ and difference $\left(K_{2}^{0}\right)$ of the neutral kaon and its antiparticle states and concluded that these particle states must have different decay modes and lifetimes. In particular they concluded that $K_{1}^{0}$ could decay to two charged pions, while $K_{2}^{0}$ would have a longer lifetime and more complex decay modes. This conclusion was based upon the conservation of $C$ in weak interaction processes.

The particle-mixing theory of Gell-Mann and Pais was confirmed in 1957 when it was demonstrated that not more than half of the particles in a beam consisting only of $K^{0}$ s decay into two charged pions. This was in spite of the incorrect assumption of $C$ invariance in weak interaction processes discovered also in 1957.

This led to a proposal by Landau [12] that the weak interactions may be invariant under the combined operation $C P$, although both $C$ and $P$ are individually maximally violated. Landau's suggestion implied that the Gell-Mann and Pais model of neutral kaons would still apply if the states, $K_{1}^{0}$ and $K_{2}^{0}$, were eigenstates of $C P$ with eigenvalues +1 and -1 , respectively. Since the charged pions were considered to have intrinsic parity $P=-1$, it was clear that only the $K_{1}^{0}$ state could decay to two charged pions, if $C P$ was conserved. 
The suggestion of Landau was accepted for several years since it nicely restored some degree of symmetry in weak interaction processes. However, in 1964 the surprising discovery was made by Christenson et al. [13] that the long-lived neutral kaon $\left(K_{2}^{0}\right)$ could decay to two charged pions. The observed violation of $C P$ conservation turned out to be very small $(0.2 \%)$ compared with the maximal violations of both $C$ and $P$ conservation separately. Indeed the very smallness of the observed $C P$ violation led to a variety of suggestions explaining it in a $C P$ conserving way. However, these efforts were unsuccessful and $C P$ violation in weak interactions was accepted.

\section{Basic Problem inherent in Standard Model}

The SM, in spite of its considerable success, has a basic problem: it is the classification of its twelve elementary particles, the leptons and quarks, employing a diverse scheme of additive quantum numbers, some of which are not conserved in weak interaction processes; and at the same time failing to provide any physical basis for this scheme. Except for charge, leptons and quarks are allotted different kinds of additive quantum numbers: the leptons having several lepton numbers, the quarks having baryon number, strangeness, charm, bottomness and topness, so that the SM classification scheme is non-unified. In addition, several additive quantum numbers of the SM, strangeness, charm, bottomness and topness, are not conserved in all interactions since they can undergo a change of one unit in weak interaction processes.

\section{Generation Model}

The GM of particle physics overcomes the basic problem inherent in the SM, replacing it with a much simpler and unified classification scheme of leptons and quarks.

\begin{tabular}{|llll|llll|}
\hline particle & $Q$ & $p$ & $g$ & particle & $Q$ & $p$ & $g$ \\
$\boldsymbol{v}_{\boldsymbol{e}}$ & 0 & -1 & 0 & $\boldsymbol{u}$ & $+2 / 3$ & $1 / 3$ & 0 \\
$\boldsymbol{e}^{-}$ & -1 & -1 & 0 & $\boldsymbol{d}$ & $-1 / 3$ & $1 / 3$ & 0 \\
$\boldsymbol{v}_{\boldsymbol{\mu}}$ & 0 & -1 & \pm 1 & $\boldsymbol{c}$ & $+2 / 3$ & $1 / 3$ & \pm 1 \\
$\boldsymbol{\mu}^{-}$ & -1 & -1 & \pm 1 & $\boldsymbol{s}$ & $-1 / 3$ & $1 / 3$ & \pm 1 \\
$\boldsymbol{v}_{\boldsymbol{\tau}}$ & 0 & -1 & $0, \pm 2$ & $\boldsymbol{t}$ & $+2 / 3$ & $1 / 3$ & $0, \pm 2$ \\
$\boldsymbol{\tau}^{-}$ & -1 & -1 & $0, \pm 2$ & $\boldsymbol{b}$ & $-1 / 3$ & $1 / 3$ & $0, \pm 2$ \\
\hline
\end{tabular}

Table 1: Generation Model additive quantum numbers

Table 1 displays the unified set of three additive quantum numbers, which are conserved in all interactions, for the GM [4]. The model describes all the observed transition amplitudes 
for both hadronic and leptonic processes in terms of only three conserved additive quantum numbers: charge $\mathrm{Q}$, particle number $\mathrm{p}$ and generation quantum number $\mathrm{g}$, provided the 'force' particles, mediating the various interactions, have $\mathrm{p}=\mathrm{g}=0$. Each generation of leptons and quarks has the same set of values for $\mathrm{Q}$ and $\mathrm{p}$ : the generations are differentiated by the generation quantum number, which in general can have multiple values. Essentially, particle number replaces both lepton number and baryon number of the SM, while the generation quantum number replaces the remaining quantum numbers of the SM other than charge, which is the same in both models.

The table indicates a simpler and unified classification scheme for leptons and quarks. Furthermore, all three additive quantum numbers are conserved in all interactions, unlike several of those (e.g. strangeness) allotted to quarks in the SM. The conservation of the generation quantum number in weak interactions was only achieved by making two postulates, which means that the GM differs fundamentally from the SM in two ways, corresponding to an interchange of the roles of the mass eigenstate and weak eigenstate quarks in the two models.

Firstly, the GM postulates that it is the mass eigenstate quarks of the same generation, which form weak isospin doublets: $(u, d),(c, s)$ and $(t, b)$. Thus the GM assumes that the $u, c$ and $t$ quarks interact with the $d, s$ and $b$ quarks, respectively, with the full strength of the chargechanging weak interactions mediated by the charged $W$ bosons, which have zero additive quantum numbers apart from charge, and do not interact at all with quarks belonging to another generation. This is guaranteed by the conservation of the generation quantum number.

Secondly, the GM postulates that hadrons are composed of weak eigenstate quarks rather than mass eigenstate quarks as in the SM. Purely for simplicity the following discussion is restricted to only the first two generations of quarks. In this approximation the weak eigenstate quarks are $d^{\prime}=d \cos \theta+s \sin \theta$ and $s^{\prime}=-d \sin \theta+s \cos \theta$, where $\theta$ is a mixing angle introduced by Cabibbo [14]. It should be noted that the extension of the discussion to all three generations is straightforward. In this case the quark-mixing parameters correspond to the CabibboKobayashi-Maskawa matrix elements [15], which indicate that inclusion of the third generation would have minimal effect on the present discussion.

The unified classification scheme of the GM makes feasible a composite version of the GM $[4,7]$. In the composite model the leptons and quarks are not elementary particles as in the SM but are composed of rishons and/or antirishons. In particular this model predicts that the down and strange quarks have opposite intrinsic parities. This is important since it implies that the weak eigenstate quarks, $d^{\prime}$ and $s^{\prime}$, are mixed-parity states so that pions exist in mixed-parity states.

It has recently been shown [9] that the early experiment of Chinowsky and Steinberger is inconclusive, if the pion, neutron and proton have a complex substructure as in the composite GM and that it is also compatible with the mixed-parity nature of the charged pion.

Similarly, it has been shown [10] that the recent determination [16] of the parity of the neutral pion, using the double Dalitz decay $\pi^{0}->e^{+} e^{-} e^{+} e^{-}$, is also compatible with the mixedparity nature of the neutral pion predicted by the composite GM. 


\section{Conclusion}

Recently, Morrison and Robson [8] have demonstrated that the $C P$ violation observed by Christenson et al. [13] for the neutral kaon system can be described in terms of mixed-quark states in hadrons. In the GM, within the two generation approximation, the long-lived neutral kaon exists in a $C P=-1$ eigenstate and the mixed-parity of the charged pions provides the twocharged pion system $\left(\pi^{+} \pi^{-}\right)$with a small component of a $C P=-1$ eigenstate so that the longlived neutral kaon can decay to two charged pions without $C P$ violation. Furthermore the rate of this decay relative to the decay into all charged modes is estimated accurately in terms of the Cabibbo-mixing angle. This suggests that $C P$ is not violated in the neutral kaon system.

\section{References}

[1] B.A. Robson, A generation model of the fundamental particles, Int. J. Mod. Phys. E 11 (2002) 555.

[2] B.A. Robson, Relation between strong and weak isospin, Int. J. Mod. Phys. E 13 (2004) 999.

[3] P.W. Evans and B.A. Robson, Comparison of quark mixing in the standard and generation models, Int. J. Mod. Phys. E 15 (2006) 617.

[4] B. Robson, The generation model of particle physics in Particle Physics, Ed. E. Kennedy, InTech Open Access Publisher, Rijeka, Croatia (2012) 1.

[5] K. Gottfried and V.F. Weisskopf, Concepts of Particle Physics, Vol. 1, Oxford University Press, New York (1984).

[6] B.A. Robson, The generation model and the origin of mass, Int. J. Mod. Phys. E 18 (2009) 1773.

[7] B.A. Robson, A quantum theory of gravity based on a composite model of leptons and quarks, Int. J. Mod. Phys. E 20 (2011) 733.

[8] A.D. Morrison and B.A. Robson, $2 \pi$ decay of the $K_{L}^{0}$ meson without CP violation, Int. J. Mod. Phys. E 18 (2009) 1825.

[9] B.A. Robson, Parity of charged pions, Int. J. Mod. Phys. E 20 (2011) 1677.

[10] B.A. Robson, Parity of neutral pion, Int. J. Mod. Phys. E 20 (2011) 1961.

[11] W. Chinowsky and J. Steinberger, Absorption of negative pions in deuterium: parity of pion, Phys. Rev. 95 (1954) 1561.

[12] L.D. Landau, On the conservation laws for weak interactions, Nucl. Phys. 3 (1957) 127.

[13] J.H. Christenson, J.W. Cronin, V.L. Fitch and R. Turlay, Evidence for the $2 \pi$ decay of the $K_{2}^{0}$ meson, Phys. Rev. Lett. 13 (1964) 138.

[14] N. Cabibbo, Unitary symmetry and leptonic decays, Phys. Rev. Lett. 10 (1963) 531.

[15] D.H. Perkins, Introduction to High Energy Physics, $4^{\text {th }}$ Ed., Cambridge University Press, Cambridge UK (2000).

[16] E. Abouzaid et al., Determination of the parity of the neutral pion via its four-electron decay, Phys. Rev. Lett. 100 (2008) 182001. 